\title{
ASTHMA
}

\section{Predictors of therapy resistant asthma: outcome of a systematic evaluation protocol}

\author{
L G Heaney, E Conway, C Kelly, B T Johnston, C English, M Stevenson, J Gamble
}

Thorax 2003;58:561-566

See end of article for authors' affiliations

Correspondence to: Dr L G Heaney, Regional Respiratory Centre, Level 8, Belfast City Hospital, Lisburn Road, Belfast BT9 7AB, UK:

Liam.Heaney@bch.n-i.nhs.uk

Revised version received 25 November 2002 Accepted for publication 8 April 2003
Background: It has been suggested that asthmatic subjects with persisting symptoms despite adequate maintenance therapy should be systematically evaluated to identify factors contributing to poor control. The aims of this study were to examine the prevalence of these factors in a cohort of sequentially referred poorly controlled asthmatics, and to determine if any factor or combination of factors predicted true therapy resistant asthma (TRA).

Methods: Patients were evaluated using a systematic evaluation protocol including induced sputum analysis, psychiatric assessment, ear, nose and throat examination, pulmonary function testing, high resolution CT scan of the thorax, and 24 hour dual probe ambulatory oesophageal pH monitoring; any identified provoking factor was treated. Asthma was managed according to BTS guidelines.

Results: Of 73 subjects who completed the assessment, 39 responded to intervention and 34 had TRA. Subjects with TRA had a greater period of instability, a higher dose of inhaled steroids at referral, more rescue steroid use, and a lower best percentage forced expiratory volume in 1 second (FEV, $\%$ ). Oesophageal reflux, upper airway disease, and psychiatric morbidity were common $157 \%$, 95\%, $49 \%$, respectively) but were not more prevalent in either group. Using multivariate logistic regression analysis, inhaled steroid dose $>2000 \mu \mathrm{gBD}$, previous assessment by a respiratory specialist, and initial $\mathrm{FEV}, \%$ of $<70 \%$ at referral predicted a final diagnosis of TRA.

Conclusions: In poorly controlled asthmatics there is a high prevalence of co-morbidity, identified by detailed systematic assessment, but no difference in prevalence between those who respond to intervention and those with TRA. Targeted treatment of identified co-morbidities has minimal impact on asthma related quality of life in those with therapy resistant disease.
$\mathrm{N}$ ational and international guidelines on the treatment of asthma advocate a stepwise approach, ${ }^{12}$ and most patients respond to treatment with moderate doses of inhaled corticosteroids alone or in addition to a long acting $\beta_{2}$ agonist. Approximately $5 \%$ of adult patients remain difficult to control despite high dose inhaled steroids, with persisting breakthrough symptoms and frequent exacerbations requiring systemic steroids. ${ }^{3}$ Debate exists as to the next best therapeutic intervention, ${ }^{1}$ but these patients are a cause of concern because of persistent symptoms and impairment of quality of life, continued impaired lung function, increased risk of asthma death, and adverse effects of high dose

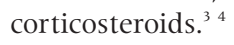

It is likely that a number of factors contribute to lack of adequate control in these subjects, including incorrect diagnosis, unrecognised systemic disease, unrecognised trigger factors (such as occupational exposures or gastrooesophageal reflux), poor compliance with treatment, and psychosocial problems. ${ }^{3-5}$ Although these factors have been proposed as contributing to poor asthma control in this population, there has been little formal documentation regarding their frequency and how targeted intervention affects outcome. Indeed, the precise relationship between symptoms, lung function, health related quality of life, and therapeutic intervention remains to be established in this group.

The ERS Task Force on Difficult Asthma identified the need for an "integrated approach to define clinical phenotypes, evaluate risk factors, understand pathophysiology and find novel therapies". ${ }^{4}$ They also suggested a period of assessment and evaluation to detect and, where possible, to modify factors leading to poor control and subsequently to identify subjects with definite therapy resistant asthma (TRA).

We hypothesised that subjects with poorly controlled asthma who had identifiable provoking factors would respond to targeted treatment of these factors and their asthma would stabilise on reduced maintenance therapy. The aim of this study was therefore to evaluate a cohort of sequentially referred poorly controlled asthmatics using a systematic evaluation protocol, and to identify factors contributing to poor control, the frequency with which they occur, and to determine if any factor or combination of factors predicted true TRA.

\section{METHODS}

Patients were recruited for protocol evaluation if they had (1) persisting refractory symptoms prompting referral to a specialist, (2) minimal maintenance therapy of long acting $\beta_{2}$ agonist and inhaled steroids ( $\geqslant 800 \mu \mathrm{g}$ beclomethasone dipropionate (BDP) or equivalent), and (3) at least one course of systemic steroids in the preceding 12 months.

Asthma was defined on the basis of typical symptoms together with current or recently documented reversibility in forced expiratory volume in 1 second $\left(\mathrm{FEV}_{1}\right)$ of $>12 \%{ }^{6}$ All recent admissions for asthma were reviewed to determine if there was objective evidence of acute severe asthma at the time of admission.

Patients were recruited to the protocol in a sequential unselected manner. Those found at visit 1 not to fulfil the definition of uncontrolled asthma outlined above were excluded at that stage. All patients were seen and assessed by the same respiratory physician at visit 1 and every subsequent clinical visit. The protocol was run on an outpatient basis and coordinated by an asthma nurse specialist.

Visit 1

At the initial visit a detailed semi-structured clinical history was recorded for all subjects and a full physical examination 
was performed. Precipitants (oesophageal reflux, upper airway disease, social difficulties, and psychiatric symptoms) were graded by the assessing physician on the basis of clinical history and graded 0 (not present/no symptoms), l (minor factor/minor symptoms), or 2 (major factor/severe symptoms).

Skin prick testing to 12 inhalant allergens, chest radiography, spirometric testing with reversibility to nebulised $\beta_{2}$ agonist, urinalysis, a standardised battery of blood tests, and induced sputum (Medix Sonix ultrasonic nebuliser, 3\% hypertonic saline) were all performed, and an asthma related quality of life questionnaire ${ }^{7}$ and the Hospital Anxiety Questionnaire $(\mathrm{HAD})^{8}$ were completed at visit 1 .

The following investigations were arranged as part of the investigative protocol: formal psychiatric interview, ear, nose and throat examination, pulmonary function testing (inspiratory/expiratory flow-volume loop, carbon monoxide transfer factor and lung volumes by helium dilution), high resolution CT scan of the thorax, 24 hour dual probe ambulatory oesophageal $\mathrm{pH}$ monitoring, and dual emission $x$ ray absorptiometry (DEXA). All subjects were offered the services of a social worker if an underlying contributory social issue was identified.

Inhaler technique was reviewed and patients were provided with the device they found most suitable and could use properly (including the provision of combination inhalers). All patients were provided with a written self-management plan, and they were given advice on the roles of precipitants in provoking their asthma symptoms and the precise role of different inhaler medications in an attempt to maximise compliance. They were instructed in keeping a peak flow record and provided with a diary. Atopic patients were provided with a standardised allergen avoidance sheet.

At visit 1 an initial management plan was instituted on clinical grounds including the requirement for a course of systemic steroids or the addition or reduction in therapy. All reviews were determined by clinical need and patients were asked to contact the clinic if their asthma deteriorated or if they had an unscheduled asthma visit or hospital admission and the details were recorded. At each review, clinical assessment was performed and spirometric parameters recorded.

\section{Management}

Asthma was managed according to BTS guidelines with treatment being stepped up and down as appropriate. ${ }^{1}$ The lowest dose of inhaled corticosteroid required to control asthma symptoms during the period of evaluation was recorded for each subject. All patients were followed up for a minimum period of 12 months after protocol assessment. If asthma symptoms were controlled, subjects were classified as having non-TRA and were discharged. TRA was defined in accordance with the ERS Task Force on Difficult Asthma as persisting symptoms due to asthma despite high dose inhaled steroids (2000 $\mu \mathrm{g}$ beclomethasone, $1600 \mu \mathrm{g}$ budesonide, $1000 \mu \mathrm{g}$ fluticasone) plus a long acting $\beta$, agonist with the requirement for either maintenance systemic steroids or at least two rescue courses of steroids in a follow up period of 12 months and despite trials of other add-on therapies e.g. leukotriene receptor antagonist or theophylline. An asthma related quality of life questionnaire and the HAD questionnaire were completed on discharge by subjects with non-TRA and 12-18 months after protocol assessment by those with TRA.

If a subject failed to attend any follow up clinic appointment a further appointment letter was sent and telephone contact was made to identify the reason for non-attendance, to establish if the patient's asthma remained stable, and to reinforce attendance at the new appointment. If the subject failed to attend for three sequential appointments no further appointments were made and the general practitioner was informed. Telephone follow up to both the subject and the primary care provider was made after 12-18 months in subjects lost to follow up to determine the outcome. When investigations or assessments suggested a course of treatment or action, this was arranged/prescribed at the time of the investigation or at the next clinical review.

Co-existent diagnoses such as bronchiectasis or unrecognised systemic disease were managed using standard interventions and treatments. If an external provoking factor was identified-for example, occupational exposure/domestic allergen-subjects were encouraged to take steps to exclude the provoking factor and the success or failure to do so was recorded. If patients gave a history of asthma symptoms consistently related to particular foods or drinks, dietetic assessment was arranged and, where appropriate, a trial period of a relevant exclusion diet (for example, sulphites, azo-dyes) instituted. If patients had an abnormal 24 hour oesophageal $\mathrm{pH}$ profile (defined in our laboratory as a distal oesophageal $\mathrm{pH}$ of $<4$ for more than $4.7 \%$ of total time ${ }^{9}$ ), a standard treatment dose of proton pump inhibitor was prescribed.

In patients with symptoms of rhinitis (itch, sneeze, rhinorrhoea, nasal obstruction) or evidence on ENT examination of reactive nasal mucosa, mucus lying in the nose/post-nasal space, a standard dose of nasal steroid \pm oral antihistamine was instituted. Other ENT diagnoses such as nasal polyps, significant inferior nasal turbinate hypertrophy, or sinusitis were treated using standard medical treatment and, if this failed, a CT scan of the sinuses was performed and surgical options considered.

Psychiatric diagnoses and treatment were determined by the psychiatrist at the time of assessment and an individual agreed treatment programme designed, involving pharmacotherapy and referral to a community psychiatric nurse, social worker, and clinical psychologist.

Dysfunctional breathlessness (atypical symptoms and exertional limitation inconsistent with pulmonary function) was managed with explanation and referral to a specialist physiotherapist for control of breathlessness/stress management and a graded exercise programme plus any additional intervention recommended after psychiatric assessment. If this did not result in a clinical response, cardiopulmonary exercise testing was arranged to quantify exercise capacity and provide physiological evidence to support a diagnosis of dysfunctional breathlessness.

Vocal cord dysfunction (VCD) was diagnosed on the basis of inspiratory stridor when symptomatic plus a combination of two of the following: abnormal inspiratory limb of the flow-volume loop, previous intubation with normal inflation pressures, or abnormal inspiratory cord motion on direct visualisation. VCD was managed with explanation and a speech and language therapy programme for control of breathlessness/voice control plus any additional intervention recommended after psychiatric assessment.

Severe chronic obstructive pulmonary disease due to chronic asthma (post-bronchodilator $\mathrm{FEV}_{1}<40 \%$ predicted) was managed with an explanation of the mechanism of breathlessness, optimisation of bronchodilator therapy and, where clinically indicated, referral to a pulmonary rehabilitation programme.

Chronic bronchitis was diagnosed with a history of daily cough and sputum production in the presence of stable asthma, normal high resolution CT scan of the thorax, and clinical and bacteriological evidence of recurrent bacterial infection.

\section{Statistical analysis}

Demographic data are presented as mean (SE) or as absolute values. Univariate analysis was performed using the unpaired $t$ test for continuous variables and $\chi^{2}$ analysis for dichotomous variables and, where appropriate, $\chi^{2}$ for trend. Logistic 
Table 1 Demographic details at referral of all subjects and those classified as therapy resistant asthma (TRA) and non-TRA after evaluation

\begin{tabular}{|c|c|c|c|c|}
\hline & All patients $(n=73)$ & TRA $(n=34)$ & Non-TRA $(n=39)$ & $\mathrm{p}$ value \\
\hline Age (years) & $42.6(1.8)$ & $43.2(2.2)$ & $42.0(2.8)$ & NS \\
\hline Female (\%) & $49(67 \%)$ & $21(62 \%)$ & $28(72 \%)$ & NS \\
\hline Age of asthma diagnosis (years) & $22.0(2.1)$ & $19.9(3.0)$ & $23.9(3.0)$ & NS \\
\hline Period of instability (months) & $53.9(8.0)$ & 75.1 (14.9) & $35.5(6.5)$ & $p<0.05$ \\
\hline Asthma duration (years) & $20.6(1.8)$ & $23.4(2.6)$ & $18.1(2.4)$ & NS \\
\hline \multicolumn{5}{|l|}{ Smoker } \\
\hline Current & 12 & 6 & 6 & NS \\
\hline Ever & 13 & 6 & 7 & NS \\
\hline Atopy $(\%)$ & $40(55 \%)$ & $21(62 \%)$ & $19(49 \%)$ & NS \\
\hline Inhaled steroid at referral ( $\mu \mathrm{g}$ BDP equivalent)* & $2347(148)$ & $2941(206)$ & $1828(173)$ & $p<0.001$ \\
\hline Rescue steroid courses in preceding 12 months & $4.3(0.4)$ & $5.3(0.7)$ & $3.7(0.4)$ & $p<0.05$ \\
\hline Maintenance systemic steroid & 21 & 16 & 5 & $p<0.01$ \\
\hline Hospital admission in preceding 12 months & $0.85(0.2)$ & $1.0(0.33)$ & $0.7(0.2)$ & NS \\
\hline Unscheduled visits in preceding 12 months $\dagger$ & $5.3(1.2)$ & $4.8(0.9)$ & $5.7(2.2)$ & NS \\
\hline Previous specialist attendance & 43 & 26 & 17 & $p<0.01$ \\
\hline Pre-bronchodilator FEV (l) & $2.1(0.10)$ & $1.90(0.14)$ & $2.27(0.15)$ & NS \\
\hline Pre-bronchodilator FEV $\%$ & $72(3)$ & $65(2)$ & $79(2)$ & $p<0.05$ \\
\hline FVC\% & $83(5)$ & $74(5)$ & $91(7)$ & NS \\
\hline $\mathrm{FEV}_{1} / \mathrm{FVC}$ ratio $\%$ & $69(2)$ & $65(2)$ & $72(2)$ & $p<0.05$ \\
\hline RV $(\%)$ & $127(5)$ & 135 (8) & 119 (8) & NS \\
\hline $\operatorname{TLC}(\%)$ & $108(2)$ & $108(3)$ & $109(3)$ & NS \\
\hline $\operatorname{TLCO}(\%)$ & $92(2)$ & $90(4)$ & $95(3)$ & NS \\
\hline \multicolumn{5}{|c|}{$\begin{array}{l}\mathrm{FEV}, \text { forced expiratory volume in } 1 \text { second; } \mathrm{FVC}=\text { forced vital capacity; } \mathrm{RV}=\text { residual volume; TLC=total lung capacity; TLCO=carbon monoxide transfer } \\
\text { factor. } \\
\text { *Bioequivalence of inhaled steroids was fluticasone }=2 \times \text { beclomethasone }=2 \times \text { budesonide. } \\
\text { tUnscheduled visits include visits to primary care and hospital accident and emergency departments. } \\
\text { Data are presented as mean (SE) or absolute values. }\end{array}$} \\
\hline
\end{tabular}

regression analysis was subsequently performed to identify the independent variables which best predicted TRA. All statistics were performed using SPSS for Windows version 11.

\section{RESULTS}

Over an 18 month recruitment period 86 patients were referred, six of whom were omitted at visit 1 because they did not fulfil the entry criteria at the time of first attendance (four were not taking a long acting $\beta_{2}$ agonist despite its apparent prescription and two were no longer difficult to control).

Of the remaining 80 subjects, six were lost to analysis (four were non-compliant with the protocol, one had oesophageal adenocarcinoma diagnosed during evaluation, and one emigrated). Telephone follow up of the four subjects who were non-compliant with the protocol indicated that one with a provisional diagnosis of VCD/hyperventilation had stopped all asthma treatment and remained well, and one with dysfunctional breathlessness had stopped inhaled steroid therapy but still used relief short acting $\beta_{2}$ agonist. The other two subjects (one with a prominent phobia of hospital and one with very poor compliance) followed a pattern of recurrent exacerbation and systemic steroid use managed in primary care. In one further subject a diagnosis of asthma could not be sustained after review of attendance and serial lung function. Of the remaining 73 subjects, 34 were classified as having TRA after evaluation. The demographic details of these subjects are shown in table 1 .

During evaluation eight subjects ( $11 \%)$ did not have a psychiatric assessment (five refused assessment, one lost referral, one already attending clinical psychology, and one failed to

Table 2 Clinical, asthma quality of life (QoL), anxiety and depression scores, and blood parameters at presentation in all subjects and those with therapy resistant asthma (TRA) and non-TRA after evaluation

\begin{tabular}{|c|c|c|c|c|}
\hline & All patients $(n=73)$ & TRA $(n=34)$ & Non-TRA $(n=39)$ & $\mathrm{p}$ value \\
\hline $\begin{array}{l}\text { Upper airway symptoms } \\
(\% \text { scoring } 0,1,2)^{*}\end{array}$ & $32,57,11$ & $32,47,21$ & $30,67,3$ & NS \\
\hline $\begin{array}{l}\text { Social problem } \\
(\% \text { scoring } 0,1,2)^{*}\end{array}$ & $63,26,11$ & $65,32,3$ & $61,21,18$ & NS \\
\hline $\begin{array}{l}\text { Psychological problem } \\
(\% \text { scoring } 0,1,2)^{*}\end{array}$ & $53,32,15$ & $59,35,6$ & $49,28,23$ & NS \\
\hline $\begin{array}{l}\text { Reflux symptoms } \\
(\% \text { scoring } 0,1,2)^{*}\end{array}$ & $27,44,29$ & $24,44,32$ & $31,43,26$ & NS \\
\hline Total clinical score (median, range)* & $3(0-6)$ & $3(0-5)$ & $3(0-6)$ & NS \\
\hline Asthma QoL score (at baseline)† & $3.3(0.14)$ & $3.3(0.22)$ & $3.2) 0.17)$ & NS \\
\hline Anxiety score $\ddagger$ & $10.6(0.6)$ & $10.6(0.7)$ & $10.7(0.9)$ & NS \\
\hline Depression scoref & $7.3(0.5)$ & $7.3(0.7)$ & $7.2(0.9)$ & NS \\
\hline Total lgE (kU/I) & $257(46)$ & $347(90)$ & $179(33)$ & NS \\
\hline Sputum eosinophils (\%) & $9(3)$ & $12(6)$ & $6(3)$ & NS \\
\hline Blood eosinophils $\left(\times 10^{9} / \mathrm{I}\right)$ & $0.41(0.05)$ & $0.45(0.06)$ & $0.39(0.08)$ & NS \\
\hline
\end{tabular}

*Clinical scores were graded by the assessing physician on the basis of clinical history and graded 0 (not present/no symptoms), 1 (minor factor/minor symptoms), 2 (major factor/severe symptoms) and presented as \% subjects in individual groups.

$\dagger$ Asthma related quality of life was assessed using the Juniper scale. ${ }^{7}$ The response options for each of the 32 items are on a 7 point scale ranging from 1 (totally limited) to 7 (not at all limited); a clinically minimal significant change in overall score is 0.5 .

$\ddagger$ Anxiety and depression scores were measured using the Hospital Anxiety and Depression scale ${ }^{8}$ (scores categorised as normal 0-7, mild 8-10, moderate 11-14, severe 15-21).

Data are presented as mean (SE) or absolute values unless stated otherwise. 
Table 3 Investigation results and outcome parameters in all subjects and those with therapy resistant asthma (TRA) and non-TRA after evaluation

\begin{tabular}{|c|c|c|c|c|}
\hline & All patients $(n=73)$ & TRA $(n=34)$ & Non-TRA $(n=39)$ & $p$ value \\
\hline Additional diagnosis & 25 & 7 & 18 & $p<0.05$ \\
\hline ICD10 psychiatric diagnosis* & $32 / 65(49 \%)$ & $16 / 33(48 \%)$ & $16 / 32(50 \%)$ & NS \\
\hline Unrecognised psychiatric illness* & $27(41 \%)$ & $15(45 \%)$ & $12(38 \%)$ & NS \\
\hline \multicolumn{5}{|l|}{ ENT examination $\dagger$} \\
\hline Normal & 3 & 1 & 2 & \multirow[t]{3}{*}{ NS } \\
\hline Mucosal & 31 & 17 & 14 & \\
\hline Structural & 26 & 15 & 11 & \\
\hline Oesophageal reflux $\ddagger$ & $31 / 54$ & $17 / 29$ & $14 / 25$ & NS \\
\hline \multicolumn{5}{|l|}{ Bone density§ } \\
\hline Normal & 28 & 11 & 17 & \multirow[t]{3}{*}{$p<0.05$} \\
\hline Osteopenia & 21 & 10 & 11 & \\
\hline Osteoporosis & 10 & 8 & 2 & \\
\hline Lowest dose inhaled steroid ( $\mu g$ BDP equivalent) $\mathbb{T}$ & $1388(74)$ & $1894(31)$ & $946(88)$ & $p<0.001$ \\
\hline Maintenance steroid after evaluation & 17 & 17 & 0 & $p<0.001$ \\
\hline Best pre-bronchodilator $\mathrm{FEV}_{1} \%$ & $89(3)$ & $83(4)$ & $95(4)$ & $p<0.05$ \\
\hline Time to best $\mathrm{FEV}_{1}$ (months) & $6.15(0.7)$ & $6.3(0.9)$ & $6.0(1.0)$ & NS \\
\hline Asthma QoL score at follow up & $4.0(0.19)$ & $3.6(0.25)$ & $4.4(0.27)$ & $p<0.05$ \\
\hline Mean difference from baseline & & 0.31 & 1.05 & \\
\hline (95\% confidence interval) & & $(-0.03$ to 0.66$)$ & $(0.59$ to 1.52$)$ & \\
\hline \multicolumn{5}{|c|}{$\begin{array}{l}\text { *ICD } 10 \text { psychiatric diagnoses were assigned after psychiatric interview and included depression, generalised anxiety disorder, panic disorder, specific } \\
\text { phobia, anorexia nervosa, paranoid delusional disorder, bipolar affective disorder, acute stress reaction, post traumatic stress disorder and } \\
\text { obsessive-compulsive personality disorder. } \\
\text { †Structural abnormalities on ENT examination were septal deviation, hypertrophy of middle/inferior turbinates, and nasal polyps. } \\
\text { †Oesophageal reflux was defined as distal oesophageal } \mathrm{pH}<4 \text { for }>4.7 \% \text { of total time. } \\
\text { §Osteoporosis was defined as T score }<-2.5 \text { and osteopenia as }-1<T \text { score }<-2.5 \text { on DEXA scan. } \\
\text { TLowest dose of inhaled steroid represents the lowest dose of inhaled steroid during the follow up period. } \\
\text { Data are presented as mean (SE) or absolute values. }\end{array}$} \\
\hline
\end{tabular}

attend), although only one of the TRA group refused to attend. Thirteen (18\%) failed to attend for ENT assessment, one from the TRA group. A 24 hour oesophageal pH profile was not available in 19 subjects (26\%), five of whom were in the TRA group (eight failed to attend for the procedure, six were unable to tolerate the probe, one failed recording, and four refused the test after substantial symptomatic improvement (non-TRA group)). A high resolution CT scan was not available in 13 subjects ( $18 \%$ ), only one of whom was in the TRA group (seven refused scan after substantial clinical improvement and six failed to attend). Sputum eosinophil counts were available in only 31 of the 73 subjects (42\%); the remainder failed to expectorate an adequate sample or to tolerate the induction procedure.

The recorded period of instability was significantly longer in subjects who subsequently had TRA (table 1). They also tended to be on a higher dose of inhaled steroid at referral, had significantly more courses of systemic steroids in the previous year, and were more likely to be on maintenance systemic steroids. It is noteworthy that, of the 21 subjects on maintenance systemic steroids at referral, 11 successfully withdrew their steroids including five who subsequently did

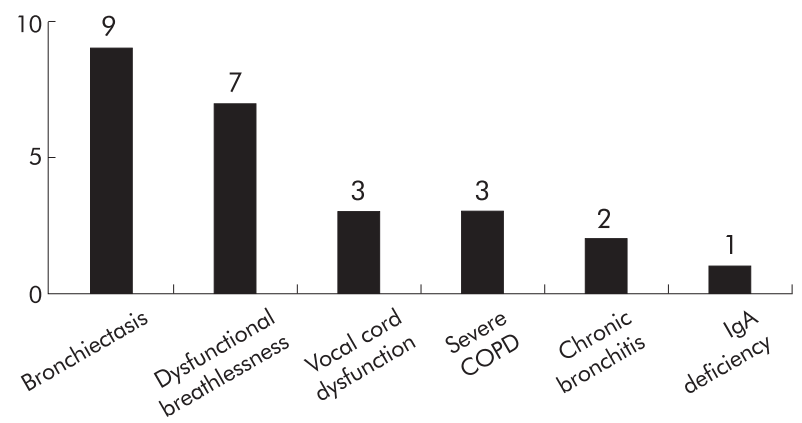

Figure 1 Additional diagnoses causing respiratory symptoms in 73 sequential referrals to a difficult asthma service (for definitions see text). Twenty five of the 73 subjects (34\%) had an additional diagnosis. not have TRA. A further seven subjects who were not on systemic steroids at referral required maintenance systemic steroids, but this group had had a median of six courses of systemic steroids in the preceding 12 months (range 2-8 courses). Subjects with TRA were more likely to have attended a respiratory specialist in the past, had lower percentage predicted $\mathrm{FEV}_{1}$, and were more obstructed at referral (table 1).

No differences were seen in individual or cumulative clinical scores for the individual groups (table 2). There was no difference in asthma related quality of life or HAD scores between the groups at baseline (table 2).

Subjects with TRA were less likely to have an additional diagnosis accounting for a proportion of their symptoms (table 3). The frequency of additional diagnoses is shown in fig l. In addition, three subjects had a history consistent with sulphite sensitivity (two had urticaria after exposure) and were given a trial exclusion diet. Gastro-oesophageal reflux, ENT pathology, and psychiatric morbidity were common but were not more prevalent in either group, although subjects with TRA were more likely to have osteoporosis (table 3). After evaluation and management, subjects with TRA were on significantly higher doses of inhaled steroids and also had a significantly lower best $\mathrm{FEV}_{1} \%$ recorded during the period of follow up (table 3). At baseline there was no difference in the asthma related quality of life scores between groups; however, a significant and clinically relevant increase was seen in those with therapy responsive asthma but not in the TRA group (table 3).

Using multivariate logistic regression analysis, the following five variables at presentation were identified as being of potential prognostic significance (using a $\mathrm{p}$ value of $<0.1$ ): higher dose of inhaled steroid, previous specialist attendance, higher total IgE, lower FEV $\%$ predicted, and a longer recorded period of instability. These variables were subsequently dichotomised and the following contrasts considered: inhaled steroid dose $>2000 \mu \mathrm{g}$ BDP equivalent, previous specialist referral, total $\mathrm{IgE}>120 \mathrm{kU} / \mathrm{l}, \mathrm{FEV} \%<70 \%$ predicted, and period of instability $>24$ months. This did not result in any loss of fit, as measured by the $\chi^{2}$ test, so dichotomy did not result in a weaker model. The final model selection was parsimonious (table 4 , cut off $\mathrm{p}<0.05$ ) and was used to generate 
Table 4 Potential prognostic factors at presentation for having therapy resistant asthma (TRA) after variables were dichotomised using logistic regression analysis

\begin{tabular}{ll}
\hline & Odds ratio $(95 \% \mathrm{Cl})$ \\
\hline Inhaled steroid $>2000 \mu \mathrm{g}$ at referral & $8.5(2.2$ to 33.1$)$ \\
Previous specialist attendance & $4.4(1.3$ to 15.0$)$ \\
Pre-bronchodilator $\mathrm{FEV}_{1} \%<70 \%$ predicted & $4.0(1.2$ to 13.9$)$ \\
\hline
\end{tabular}

\begin{tabular}{|c|c|c|c|}
\hline $\begin{array}{l}\text { Inhaled steroid } \\
>2000 \mu \mathrm{g} \mathrm{BDP} \\
\text { equivalent }\end{array}$ & $\begin{array}{l}\text { Presenting FEV } \\
<70 \% \text { predicted }\end{array}$ & $\begin{array}{l}\text { Previous } \\
\text { specialist } \\
\text { referral }\end{array}$ & $\begin{array}{l}\text { Probability of } \\
\text { TRA }\end{array}$ \\
\hline$x$ & $x$ & $x$ & 0.08 \\
\hline$x$ & $x$ & $r$ & 0.27 \\
\hline$x$ & $r$ & $x$ & 0.29 \\
\hline$r$ & $x$ & $x$ & 0.44 \\
\hline$x$ & $r$ & $r$ & 0.62 \\
\hline$r$ & $x$ & $\nu$ & 0.76 \\
\hline$r$ & $r$ & $x$ & 0.78 \\
\hline$\nu$ & $r$ & $\nu$ & 0.93 \\
\hline
\end{tabular}

estimates of probability of having TRA with different prognostic combinations at presentation (table 5).

\section{DISCUSSION}

This study shows that there is significant co-morbidity in a population of unselected sequentially referred asthmatics with persisting refractory symptoms. A systematic evaluation protocol allows recognition and management of these co-morbidities and identification of a population of asthmatics with true therapy resistant disease. However, our initial hypothesis that patients with TRA would have fewer identifiable co-morbidities amenable to treatment was not supported because we were unable to identify any difference in co-morbidities (other than osteoporosis) between the groups.

Therapy resistant disease was defined on the basis of clinical assessment and treatment requirements. This differentiation is supported by the asthma quality of life data where there was no difference at baseline between the groups, but those defined as having a therapeutic response had significantly higher and clinically relevant improvements in their asthma related quality of life scores after evaluation and treatment. ${ }^{10}$ It is also noteworthy that, in the TRA group, despite systematic and detailed assessment of provoking factors and meticulous management of their asthma, asthma quality of life scores did not show any clinically relevant change. This suggests that, if TRA is present, management of identified co-morbidity will have little impact on disease specific quality of life.

Only one previous systematic evaluation protocol for patients with poorly controlled asthma has been published. ${ }^{11}$ This protocol evaluated 42 patients over an 8 year period from 1982 to 1990 and concluded that, after working through the protocol, $74 \%$ were no longer difficult to control which suggests that protocol guided care improves outcome both in terms of lung function and patient symptoms. In that study one of the most useful interventions was commencing patients on inhaled corticosteroids, which reflects asthma management at the time but also suggests that their subjects had mild to moderate asthma.
As anticipated, during the protocol there were a number of failures to attend and pre-agreed withdrawals from psychiatric and ENT assessments, CT chest scans, and oesophageal pH monitoring, although this was minimal in the therapy resistant group. Because of this, we believe that failure to have these assessments is unlikely to have reclassified any of the patients and would not alter the conclusions from this observational study. The pre-agreed attendance failures reflected a response to achieving better symptom control and a decision that additional investigation and assessment was not required. We believe the unplanned failures to attend for assessments are also indicative of symptomatic improvement and a decision by the subject that further evaluation was not required.

There were a number of demographic features present at referral which could be used to identify subjects with TRA. Subjects were more likely to record poor asthma control for a longer period of time compared with subjects whose disease responded to targeted intervention. They were also more likely to have been previously assessed by a respiratory specialist, which suggests a prior period of refractory disease. While we did not establish if their disease had previously stabilised and then relapsed or remained unstable, the observations taken together suggest that the pattern of therapy resistant disease, once evolved, tends to persist with time.

In addition, patients with TRA had both lower initial $\mathrm{FEV}_{1} \%$ and lower best $\mathrm{FEV}_{1} \%$, consistent with a greater degree of fixed airflow obstruction and airways remodelling which does not relate to the presence of previous or current smoking. This is consistent with previous studies showing significantly reduced lung function in subjects with severe asthma. ${ }^{12}{ }^{13}$ As our study was cross sectional, it is impossible to determine whether worse lung function causes TRA or vice versa. Given the prolonged period of instability and the fact that there was no difference in the duration of asthma, these observations suggest that unstable disease (which could be regarded as a surrogate of uncontrolled or a particular type of therapy resistant inflammation) is associated with progressive remodelling and an accelerated decline in lung function.

Sputum eosinophilia at presentation was not related to the presence of TRA. We believe that this is because an initial sputum eosinophilia is likely to reflect both subjects with relatively refractory inflammation and other subjects who are variably compliant at presentation. A previous cross sectional study has shown a relationship between sputum eosinophilia and the degree of fixed airflow obstruction in subjects with severe asthma. ${ }^{12}$ There was a trend towards higher eosinophil counts in subjects with lower post-bronchodilator FEV $\%$ (data not shown) but this was not significant. The failure of a large number of subjects to tolerate the sputum induction procedure or to produce an adequate sample may also be relevant as this may select a subgroup of asthmatics within the overall group.

Subjects with TRA were taking significantly higher doses of inhaled steroids at referral than those with non-TRA. The mean dose in the TRA group was above the maximal recommended dose for inhaled steroids ${ }^{1}$ and probably reflects persisting failure to respond to treatment over time and a gradual increase in anti-inflammatory medication in an attempt to gain control. Given that the dose response curve for inhaled steroids is relatively flat at higher doses with increased side effects due to systemic bioavailability, it is probable that some of these subjects were receiving inappropriately high doses of inhaled steroids. ${ }^{14}$

Patients with TRA also received more rescue courses of steroids in the preceding year and were more likely to be on maintenance systemic steroids at referral, consistent with more severe and relatively steroid dependent disease. It is notable, however, that five of 21 (24\%) subjects who were on systemic steroids at referral did not have TRA and strongly supports early referral of subjects requiring maintenance systemic steroids for specialist assessment. 
The large doses of inhaled steroids and frequent systemic steroids are consistent with the observation that osteoporosis was significantly more prevalent in the TRA group. It is also consistent with the greater period of instability during which, based on the 12 month pre-referral period, they probably would have had further significant exposure to systemic steroids. It is interesting that the duration of asthma did not differ between the groups, which is consistent with the suggestion that it is the higher cumulative steroid exposure that occurs during unstable disease which causes premature loss of bone density.

As anticipated, identifying a diagnosis in addition to asthma which contributed to symptoms was associated with non-TRA. In addition, prior assessment by another respiratory specialist was associated with TRA, although this did not appear to be related to identification of an additional diagnosis (those with prior specialist assessment included 16 of the 25 subjects assigned an additional diagnosis). There was also no relationship with any particular individual additional diagnosis.

The logistic regression analysis defined a number of demographic variables which appeared to be of prognostic significance. We believe the three variables in the final model (inhaled steroid dose $>2000 \mu \mathrm{g}$, pre-bronchodilator $\mathrm{FEV}_{1}$ of $<70 \%$ on this dose of inhaled steroid, and previous respiratory specialist referral) reflect relative steroid resistance and chronic unstable disease. It is interesting that a prebronchodilator $\mathrm{FEV}_{1}$ of $<70 \%$ at presentation despite high dose inhaled steroids and long acting $\beta_{2}$ agonist predicted resistance to treatment. In the TRA group the prebronchodilator $\mathrm{FEV}_{1} \%$ at presentation was significantly lower than the best pre-bronchodilator $\mathrm{FEV}_{1} \%$ during follow up, suggesting that it is the inability to maintain lung function at a certain level despite intensive treatment rather than the degree of fixed airflow obstruction that defines therapy resistant disease. In support of this, we have shown that the loss of relationship between objective measures of asthma control and $\mathrm{FEV}_{1} \%$ in patients with severe asthma is explained by patients with fixed airflow obstruction (data not shown). In this group, fixed airflow limitation presumably generates exertional symptoms only, but other features of poor asthma control such as night time symptoms are not problematic.

From table 5 it can be seen that any combination of these three variables at presentation produces a range of prognostic probabilities as to whether a patient has TRA. Thus, if a patient is on $>2000 \mu \mathrm{g}$ inhaled steroid, has a pre-bronchodilator $\mathrm{FEV}_{1}$ of $<70 \%$ on this dose of inhaled steroids, and has previously attended a respiratory specialist, the chance of that patient having TRA is $93 \%$. Similarly, if none of these factors is present, the chance of having TRA falls to $8 \%$. The final model is simple and easy to apply and the variables are easily ascertained at presentation. We are currently evaluating this model prospectively in a separate cohort to determine negative and positive predictive values.

In summary, we have evaluated in detail a cohort of sequentially referred patients with difficult to control asthma and have identified significant co-morbidity in this population. Over $50 \%$ of those evaluated subsequently did not have
TRA, supporting the use of a detailed and systematic evaluation of this population. In contrast to our original hypothesis, we did not identify fewer treatable provoking factors in the TRA group, but treatment of identified comorbidities had no impact on asthma related quality of life in this group. We have identified a number of demographic features at presentation which are associated with the presence of TRA, and developed a simple model which will define patients with TRA at presentation. We are currently evaluating the predictive value of this model in a further cohort of patients.

Authors' affiliations

L G Heaney, C English, J Gamble, Regional Respiratory Centre, Belfast City Hospital, Belfast, UK

L G Heaney, B T Johnston, Department of Medicine, Queens

University, Belfast, UK

E Conway, C Kelly, Department of Mental Health, Queens University, Belfast

M Stevenson, Department of Epidemiology and Statistics, Queens

University, Belfast

\section{REFERENCES}

1 British Thoracic Society/Scottish Intercollegiate Guidelines Network. British guideline on the management of asthma. Thorax 2003;58 (Suppl I):i 1-94.

2 Global Initiative for Asthma. Global strategy for asthma management and prevention. NIH publication no. 02-3659. National Institutes of Health. National Heart Lung and Blood Institute, 2002

3 Barnes PJ, Woolcock AJ. Difficult asthma. Eur Respir J 1998;12:1209-18.

4 Chung KF, Godard P, Adelroth E, et al. Difficult/therapy-resistant asthma: the need for an integrated approach to define clinical phenotypes, evaluate risk factors, understand pathophysiology and find novel therapies. European Respiratory Society Task Force on Difficult/Therapy-Resistant Asthma. Eur Respir J 1999;13:1198-208.

5 Proceedings of the ATS Workshop on Refractory Asthma. Current understanding, recommendations, and unanswered questions. Am J Respir Crit Care Med 2000;162:2341-51.

6 Quanjer PH, Tammeling GJ, Cotes JE, et al. Lung volumes and forced ventilatory flows. Report Working Party Standardization of Lung Function Tests, European Community for Steel and Coal. Official Statement of the European Respiratory Society. Eur Respir J Suppl 1993;16:5-40.

7 Juniper EF, Guyatt GH, Ferrie PJ, et al. Measuring quality of life in asthma. Am Rev Respir Dis 1993;147:832-8.

8 Zigmond AS, Snaith RP. The hospital anxiety and depression scale. Acta Psychiatr Scand 1983;67:361-70.

9 Johnston BT, McFarland RJ, Collins JS, et al. Symptom index as a marker of gastroesophageal reflux disease. Br J Surg 1992;79:1054-5.

10 Juniper EF, Guyatt GH, Willan A, et al. Determining a minimal important change in a disease-specific quality of life questionnaire. J Clin Epidemiol 1994;47:81-7

11 Irwin RS, Curley FJ, French CL. Difficult-to-control asthma. Contributing factors and outcome of a systematic management protocol. Chest 1993; 103:1662-9.

12 Ten Brinke A, Zwinderman A, Sterk P, et al. Factors associated with persistent airflow limitation in severe asthma. Am J Respir Crit Care Med 2001:164:744-8.

13 Little SA, MacLeod KJ, Chalmers GW, et al. Association of forced expiratory volume with disease duration and sputum neutrophils in chronic asthma. Am J Med 2002;1 12:446-52.

14 Barnes PJ, Pedersen S, Busse WW. Efficacy and safety of inhaled corticosteroids: new developments. Am J Respir Crit Care Med $1998 ; 157: S 1-53$ 